\title{
Intravascular large B-cell lymphoma with diffuse ground glass lesion on chest computed tomography diagnosed using transbronchial lung cryobiopsy: a case report
}

\author{
Dongyi Zhu ${ }^{1 \#}$, Yan Shen ${ }^{2 \#}$, Jianhao Huang ${ }^{1}$, Liming Zhao ${ }^{1}$ \\ ${ }^{1}$ Department of Respiratory Medicine, Shanghai East Hospital, Tongji University School of Medicine, Shanghai, China; ${ }^{2}$ Department of Emergency \\ Medicine, Shanghai East Hospital, Tongji University School of Medicine, Shanghai, China \\ \#These authors contributed equally to this work. \\ Correspondence to: Liming Zhao. Department of Respiratory Medicine, Shanghai East Hospital, Tongji University School of Medicine, Shanghai \\ 200123, China. Email: 1976zlm@163.com.
}

\begin{abstract}
A 68-year-old man was presented with high fever of unknown origin for 3 weeks and nonproductive cough for 1 week. A chest computed tomography (CT) scan revealed multiple nodules and ground glass opacities (GGO) in both lungs. The patient was initially diagnosed with hypersensitivity pneumonitis based on the result of bronchoalveolar lavage fluids (BALF). After treatment with methylprednisolone for 2 weeks, the patient's fever recurred, with no resolution of lesions on chest CT. The patient consented to positron emission tomography (PET)/CT. It showed that fluorodeoxyglucose (FDG) metabolism was significantly increased in the spleen, whole skeleton, and both lungs, suggesting a malignant hematological disease. Large B-cell lymphoma was diagnosed by bone marrow puncture and flow cytometry. Transbronchial lung cryobiopsy was performed to evaluate the diffuse lung lesion. Hematoxylin-eosin (HE) staining showed diffuse infiltration of heterotypic cells in the pulmonary interstitial capillaries. Furthermore, immunohistochemical examination results suggested lung infiltration of B lymphohematopoietic system tumors. The patient was finally diagnosed as intravascular large B-cell lymphoma (IVLBCL). IVLBCL with diffuse lung ground glass lesions is very rare and difficult to diagnose. Transbronchial lung cryobiopsy, as an emerging procedure, plays an important role in the diagnosis of interstitial lung disease and has gained popularity for a lower complication rate and acquisition of more tissue samples.
\end{abstract}

Keywords: Intravascular large B-cell lymphoma (IVLBCL); cryobiopsy; diffuse lung disease; case report

Submitted May 04, 2021. Accepted for publication Jul 16, 2021.

doi: $10.21037 /$ tcr-21-769

View this article at: https://dx.doi.org/10.21037/tcr-21-769

\section{Introduction}

Intravascular large B-cell lymphoma (IVLBCL) is a rare hematological malignancy, which belongs to extranodal large B-cell lymphoma. It selectively grows within the lumina of the small vessels (1). When lymphoma cells proliferate within vessels, there will be some clinical symptoms presenting prominent neurologic, hematologic, skin, bone marrow, and pulmonary involvement (2). It is difficult to make a definite diagnosis of pulmonary IVLBCL because the clinical manifestation and imaging features are often nonspecific. Bilateral ground glass opacities
(GGO) are the most common pattern of chest computed tomography (CT) findings of IVLBCL (2). However, the differential diagnoses of pulmonary GGO include many etiologies, not only malignant tumor but also infection and interstitial inflammatory diseases. Furthermore, although pulmonary involvement is found commonly in this disease at autopsy, initial presentation in the lungs is uncommon. Here, we describe the case of a 68 -year-old patient with fever and diffuse ground glass lesions on chest CT scan, which was finally diagnosed as IVLBCL using transbronchial lung cryobiopsy. We present the following 

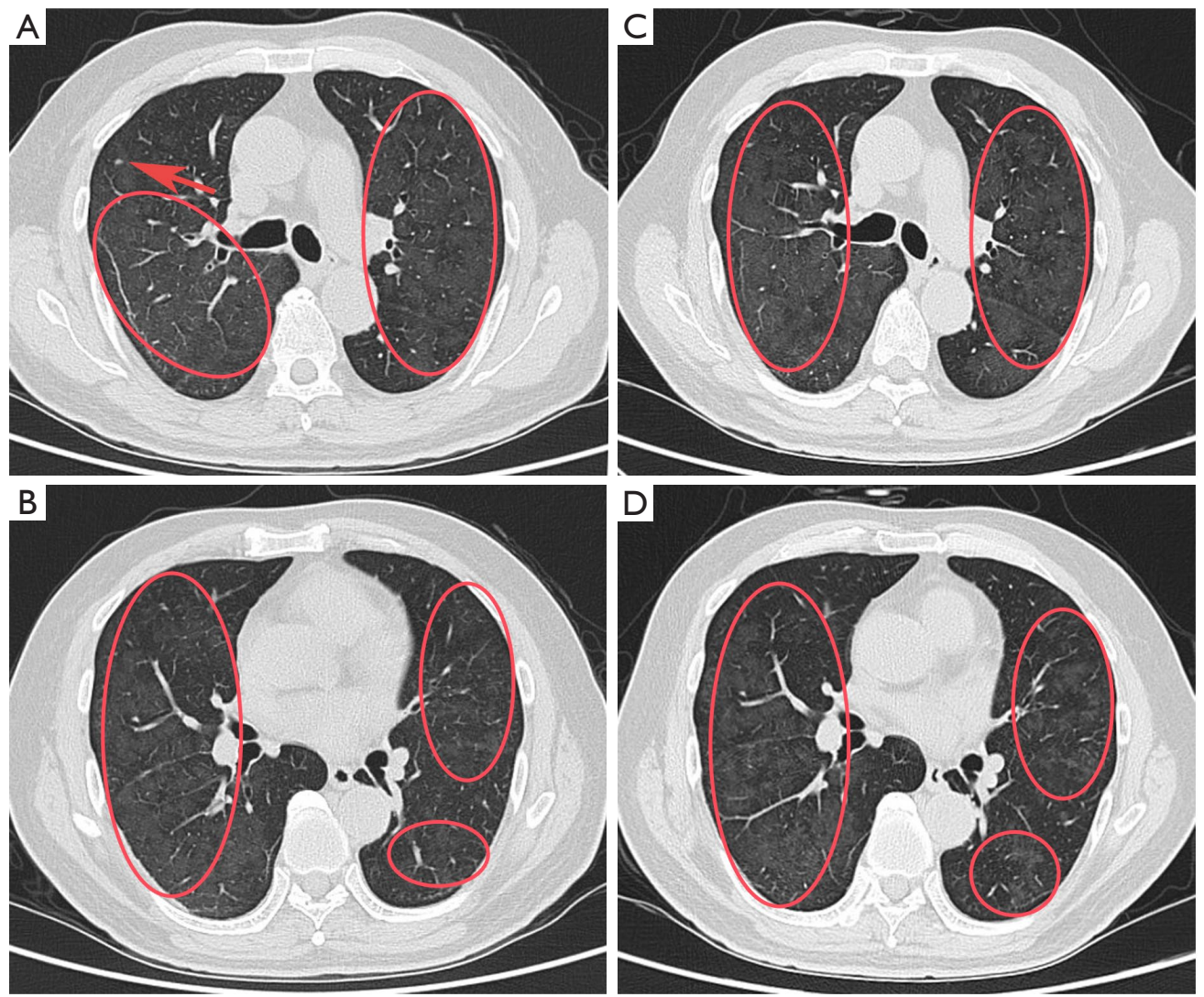

Figure 1 Imaging of the lung. (A,B) Chest CT demonstrating diffuse ground-glass infiltrates throughout the lungs bilaterally when the patient was admitted (red arrow: nodule; oval: GGO). (C,D) Chest CT demonstrating diffuse ground-glass infiltrates throughout the lungs bilaterally after the treatment with methylprednisolone for nearly 2 weeks (oval: GGO). CT, computed tomography; GGO, ground glass opacities.

article in accordance with the CARE reporting checklist (available at https://dx.doi.org/10.21037/tcr-21-769).

\section{Case presentation}

All procedures performed in studies involving human participants were in accordance with the ethical standards of the institutional and/or national research committee(s) and with the Helsinki Declaration (as revised in 2013). Written informed consent was obtained from the patient for publication of this case report and accompanying images. A copy of the written consent is available for review by the editorial office of this journal. The patient was a 68-year-old man who presented with intermittent fever for 3 weeks and non-productive cough for 1 week. The body temperature fluctuated from 37.4 to $39.0^{\circ} \mathrm{C}$, with fluctuations mainly occurring in the afternoon or at night. Laboratory tests showed normal routine blood results (white blood cell count: $5.86 \times 10^{9} / \mathrm{L}$, eosinophil count: $0.01 \times 10^{9} / \mathrm{L}$, hemoglobin concentration: $132 \mathrm{~g} / \mathrm{L}$, platelet count: $179 \times 10^{9} / \mathrm{L}$ ) and an increased C-reactive protein (CRP) level $(50 \mathrm{mg} / \mathrm{dL})$. A chest $\mathrm{CT}$ showed multiple nodules and GGO in both lungs (Figure 1A,1B). Owing to the global spread of corona virus disease 2019 (COVID-19), the virus nucleic acid and antibody tests were performed and the results were negative.

Before admission, the patient was treated with azithromycin and cefoperazone-sulbactam for 5 days at the emergency department with no effect. The patient had a medical history of psoriasis, which was treated with a combination of oral and external glucocorticoids for many years. But he had stopped taking oral drugs for a long time with the stabilization of the disease.

After admission, examination revealed that physical findings were almost normal except for partial desquamation of the skin, with no enlarged lymph nodes or neurological 


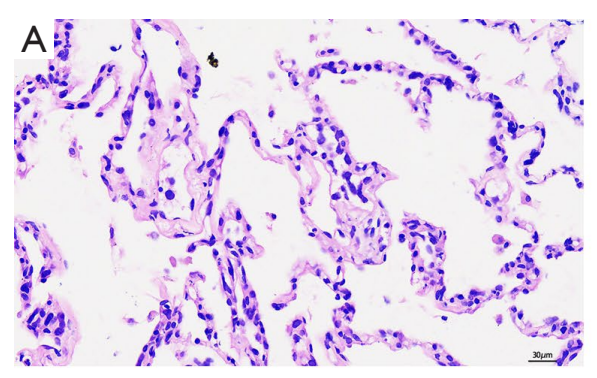

$\mathrm{HE}$

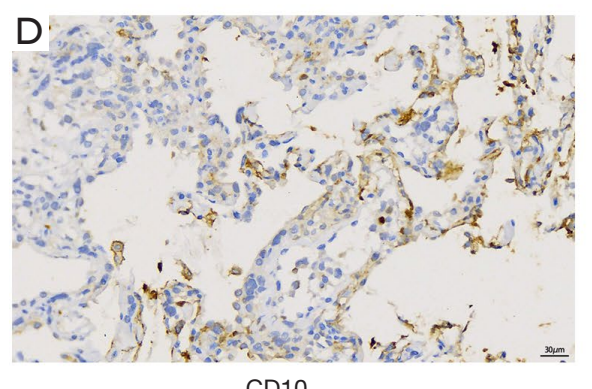

CD10

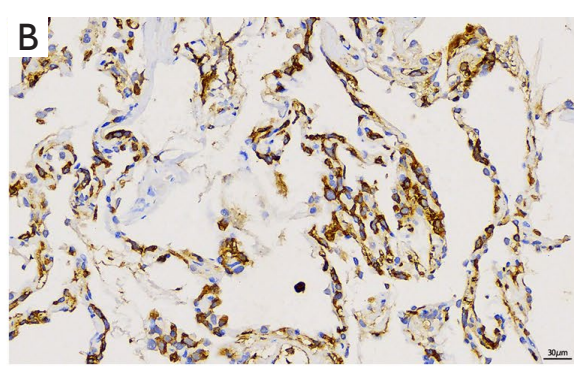

CD19

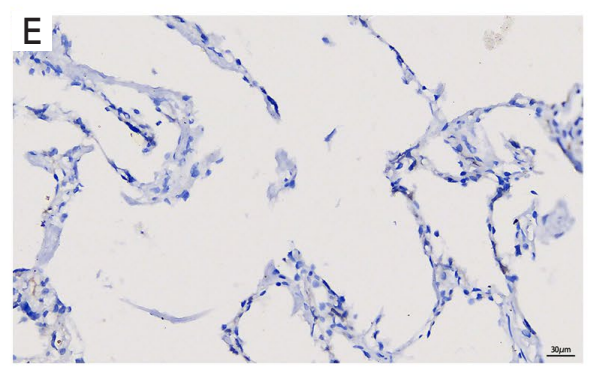

MUM1

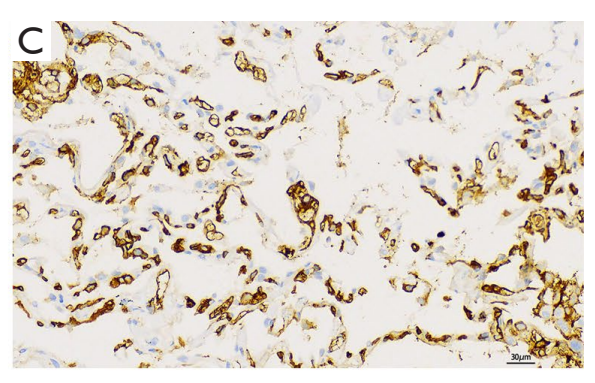

CD20

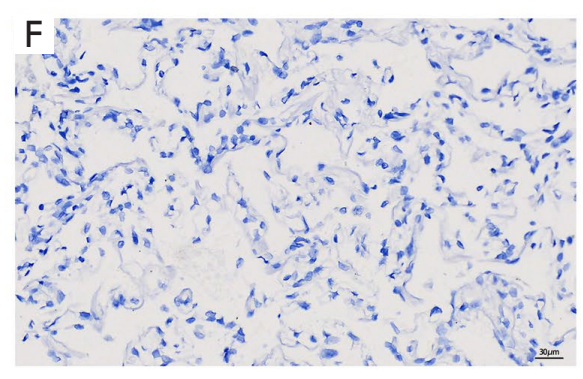

EBER

Figure 2 Histology of the lung. (A) A HE stained transbronchial lung cryobiopsy showing diffuse infiltration of heterotypic cells in the pulmonary interstitial capillaries (original magnification $\times 400$ ). (B) IHC stain showing CD19-positive tumor cells in the lumina of small capillaries (original magnification $\times 400$ ). (C) IHC stain showing CD20-positive tumor cells in the walls of small capillaries (original magnification $\times 400$ ). (D) IHC stain showing CD10-negative tumor cells in the walls of small capillaries (original magnification $\times 400$ ). (E) IHC stain showing MUM1-negative tumor cells in the walls of small capillaries (original magnification $\times 400)$. (F) IHC stain showing EBERnegative tumor cells in the walls of small capillaries (original magnification $\times 400$ ). HE, hematoxylin-eosin; IHC, immunohistochemistry; EBER, Epstein Barr virus-encoded small nuclear early region.

signs. Blood gas analysis was normal in room air. The patient consented to a bronchoalveolar lavage operation, and the bronchoalveolar lavage fluids (BALF) were collected for microbiological examinations and metagenomic next generation sequencing (mNGS). The total cell count of the BALF was $157,000 / \mathrm{mL}$. The cells consisted of macrophages (24\%), lymphocytes (62\%), neutrophils (12\%), and ciliated cells (2\%). Cultures and mNGS of the BALF were negative for bacterial, fungal, and mycobacterial pathogens. The evaluation for autoimmune diseases was negative, including rheumatoid factor, antinuclear antibodies, and C- and $\mathrm{P}$-antineutrophil cytoplasmic antibodies. Examinations for a broad range of serum tumor markers were all negative. Allergen inhalation showed that dust mite was positive with mildly elevated immunoglobulin E (185 IU/mL). Hypersensitivity pneumonitis was clinically diagnosed. However, there was no resolution of diffuse GGO on chest CT after methylprednisolone $40 \mathrm{mg}$ daily for 2 weeks (Figure 1C,1D). Meanwhile, the patient developed fever again.

Further examination was performed. Due to a sustained increase in lactic dehydrogenase $(1,220 \mu / \mathrm{L})$, ferritin $(>2,000 \mathrm{ng} / \mathrm{mL})$ and d-dimer $(10.3 \mathrm{mg} / \mathrm{L})$, malignant tumor was highly suspected. The patient consented to positron emission tomography (PET)/CT and transbronchial lung cryobiopsy. PET/CT showed that fluorodeoxyglucose (FDG) metabolism was significantly increased in the spleen, whole skeleton, and both lungs, suggesting a malignant hematological disease. Hematoxylin-eosin (HE) staining showed diffuse infiltration of heterotypic cells in the pulmonary interstitial capillaries. Immunohistochemical examination results showed CD20 (+), CD19 (+), Pax$5(+)$, CD3 (-), ck-pan (epithelial +) and kappa (+), suggesting lung infiltration of B lymphohematopoietic system tumors. It was further illustrated germinal centre B-cell like (GCB) phenotype from the result of CD10 $(-)$, bcl-6 (+) and MUM1 (-) according the decision tree proposed by Hans et al. (Figure $2 A-2 E$ ) (3). It was not common, but a minority of IVLBCL patients were GCB phenotype (4). The results of bone marrow puncture showed that bone marrow hyperplasia was active and the ratio of grain to red decreased (1.09:1). The granulocytic 
hyperplasia was active with left nuclear shift. The erythroid hyperplasia was active with karyolobism. The megakaryocytic hyperplasia was active, totally with 34 megakaryocytes. It also showed $10.5 \%$ abnormal lymphocytes. They were large and irregular with hemophagocytic cells visible. Flow cytometry results showed that $1.0 \%$ of CD5 (+) and CD10 (-) monoclonal B lymphocytes were found. The forward scatter signal was enhanced and located in monocytes. Immunophenotypes of CD19 (++), CD5 (+), CD20 (+), fmc7 (+), CD10 (-), CD23 $(-)$ and kappa light chains in the cell membrane showed restricted expression, which was same as in the lung, suggesting abnormal monoclonal B cells. Furthermore, the patient was positive for Epstein Barr virus (EBV), capsid antigen (CA) and nuclear antigen (NA) IgG antibodies, which illustrated that he had a history of EBV infection. Furthermore, the result of in situ hybridization using EBVencoded small nuclear early region (EBER) was negative, which revealed that EBV infection was not relative with IVLBCL in this case (Figure $2 F$ ).

The patient was eventually diagnosed with IVLBCL. However, the patient developed progressive leukopenia, erythrocytopenia and thrombocytopenia, which was considered as hemophagocytic syndrome. Unfortunately, the patient declined further treatment and left the hospital.

\section{Discussion}

IVLBCL is a distinct type of extranodal diffuse large B-cell lymphoma. The main characteristic is that lymphoma cells selectively proliferate within the lumen of small blood vessels (5). The clinical presentation of IVLBCL is highly variable, making it difficult to diagnose. The main clinical manifestation included fever, neurological symptoms, hypoxemia, skin eruption, anemia (hemoglobin concentration $<110 \mathrm{~g} / \mathrm{L}$ ), thrombocytopenia, hypoalbuminemia, and splenomegaly (5). When lungs are involved in IVLBCL, there are some respiratory symptoms including cough, sputum production, and dyspnea. Moreover, some patients also present with hypoxemia, pulmonary embolism or hemophagocytic syndrome (6).

In this case, our patient had few symptoms except fever and diffuse GGO on chest imaging. Methylprednisolone was administered for 2 weeks according to the suspected diagnosis of hypersensitivity pneumonitis, but the patient's fever recurred with no resolution of lesions on chest CT. Fever of unknown origin is by far the most common systemic symptom of IVLBCL, while initial presentation with pulmonary symptoms is rare. The lung appearance on chest CT varies, with findings including interlobular septal thickening, centrilobular nodules and GGO. Interstitial shadows and thickening of bronchovascular bundles usually suggest hematological and/or lymphatic spread (2). Matsue et al. analyzed the clinical data of 42 IVLBCL patients diagnosed over the past 20 years, and pleural effusion was detected in 15/42 (40.0\%) patients and GGO was detected in $9 / 42(21.4 \%)$ on chest CT (5). The study did not further distinguish the distribution, quantity, and characteristics of GGO. Some studies reported that the CT findings of IVLBCL presented as patchy, subpleural or diffused GGOs. Some other uncommon CT findings included bilateral centrilobular and peribronchial GGOs (7).

The mechanism of selective intravascular location of IVLBCL is largely unknown. The adhesion interaction between lymphocyte cell-surface receptors and components of the vascular endothelium and the extracellular matrix provide a vital role in regulating lymphocyte migration and homing. Ponzoni et al. analyzed clinical and immunohistologic data on surgical specimens from six cases of IVLBCL and found that all the cases were consistently negative for CD29 and CD54. However, CD29 and CD54 are key molecules for transvascular trafficking and migration. Their consistent absence in IVLBCL may contribute to their inability to extravasate (8). Kato et al. reported that CXCR3 expression in the IVLBCL patient was in the atypical lymphocytes, while its ligand, CXCL9, was detected in blood vessels, providing a possible new clue to the pathogenesis of IVLBCL by virtue of the characteristic expression of CXCL9-CXCR3 (9).

FDG-PET has been regarded as a valuable procedure for the diagnosis of IVLBCL. It has a known role in the assessment of diffuse large B-cell lymphoma, both at the time of diagnosis and in monitoring response to treatment. FDG-PET is sensitive in the organs with rich blood supply, specifically the lungs and kidneys (10). In this case, when treatment with methylprednisolone had no effect on the patient, FDG-PET was performed for fever of unknown origin and assisted in the preliminary diagnosis. TobinVealey et al. analyzed 3 IVLBCL patients and reported that FDG-PET scans could help to support a diagnosis of IVLBCL when clinical suspicion is high. Further, clinicians might be able to use the imaging results to guide an appropriate site for biopsy to confirm diagnosis (11).

Histopathology is the gold standard in the diagnosis of interstitial lung diseases. Surgical lung biopsy in video thoracoscopy can obtain the adequate tissue. 
However, many patients with interstitial lung diseases have comorbidities and cannot endure the surgery. It will significantly increase the incidence of complications. Colella et al. reported that surgical lung biopsies were associated with a significant mortality at 90 days, mainly for acute exacerbation of idiopathic pulmonary fibrosis (12). Traditional transbronchial lung biopsy (TBLB) is useful in subjects with high resolution computed tomography (HRCT) scan documenting alveolar opacification, bronchocentric nodules, or perilymphatic lines/nodules. It can be easily sampled with TBLB when the disorders are centered around terminal and respiratory bronchioles or are distributed along the lymphatic routes (12). Though TBLB is optional in the diagnosis of lymphoproliferative diseases, it cannot provide a sufficient amount of tissue for complete immunohistochemistry (IHC)/molecular analysis, and samples are often crushed by artefact (13).

Transbronchial lung cryobiopsy is an emerging procedure to obtain lung tissue for the diagnosis of interstitial lung disease and has gained popularity because it is less invasive and has a lower complication rate compared with nonselective surgical lung biopsy. Furthermore, it can get a large enough lung tissue samples without crushing artefact. Therefore, it improves the diagnostic rate of TBLBs in diffuse interstitial lung diseases (14). Bianchi et al. analyzed 13 cases of lymphoproliferative disorders and illustrated that 12 cases reached an accurate diagnosis by HE staining, IHC and molecular ancillary studies. In all cases, the amount of available lung tissue was sufficient for all ancillary studies. Meanwhile, there were no serious complications as severe bleeding or pneumothorax occurring in any case (15). In this case, the patient consented to transbronchial lung cryobiopsy, and lung tissues were obtained from the posterior segment of the right upper lobe and the dorsal segment of the right lower lobe. The final diagnosis was confirmed as IVLBCL by lung tissue histopathology and bone marrow aspiration.

The prognosis of IVLBCL patients will be greatly improved when they get a timely and accurate diagnosis and appropriate treatment. Cyclophosphamide, doxorubicin, vincristine, and prednisone (CHOP) or CHOP-like regimens plus rituximab is the main treatment method for IVLBCL. However, the difficulties in diagnosis often lead to delay in disease treatment (2). Hypersensitivity pneumonitis was initially diagnosed in our patient, and he received treatment with methylprednisolone for nearly 2 weeks. The patient developed hemophagocytic syndrome by the time IVLBCL was diagnosed, resulting in the loss of optimal therapy time.

\section{Conclusions}

In conclusion, IVLBCL should be considered in patients with persistent fever with diffuse lung lesions, although it is a rare disease. Transbronchial lung cryobiopsy should be conducted early for diagnostic superiority.

\section{Acknowledgments}

We thank Prof. Xianghua Yi and Xuyou Zhu from Tongji Hospital Affiliated to Tongji University for the technical support of Pathology and Prof. Qiang Li and Zhibing Luo from East Hospital Affiliated to Tongji University for the transbronchial lung cryobiopsy for the patient.

Funding: This work is supported by the Natural Science Foundation of China (Grant numbers: 82071751, 81372529); The Talent Training Plan of Shanghai East Hospital (Grant number: 2019yhrcjh05).

\section{Footnote}

Reporting Checklist: The authors have completed the CARE reporting checklist. Available at https://dx.doi. org/10.21037/tcr-21-769

Peer Review File: Available at https://dx.doi.org/10.21037/ tcr-21-769

Conflicts of Interest: All authors have completed the ICMJE uniform disclosure form (available at https://dx.doi. org/10.21037/tcr-21-769). The authors have no conflicts of interest to declare.

Ethical Statement: The authors are accountable for all aspects of the work in ensuring that questions related to the accuracy or integrity of any part of the work are appropriately investigated and resolved. All procedures performed in studies involving human participants were in accordance with the ethical standards of the institutional and/or national research committee(s) and with the Helsinki Declaration (as revised in 2013). Written informed consent was obtained from the patient for publication of this case report and accompanying images. A copy of the written consent is available for review by the editorial office of this journal.

Open Access Statement: This is an Open Access article distributed in accordance with the Creative Commons 
Attribution-NonCommercial-NoDerivs 4.0 International License (CC BY-NC-ND 4.0), which permits the noncommercial replication and distribution of the article with the strict proviso that no changes or edits are made and the original work is properly cited (including links to both the formal publication through the relevant DOI and the license). See: https://creativecommons.org/licenses/by-nc-nd/4.0/.

\section{References}

1. Satoh T, Arai E, Kayano H, et al. Pulmonary intravascular large B-cell lymphoma accompanying synchronous primary pulmonary adenocarcinoma and benign interstitial lesions. J Clin Exp Hematop 2019;59:140-4.

2. Yu H, Chen G, Zhang R, et al. Primary intravascular large B-cell lymphoma of lung: a report of one case and review. Diagn Pathol 2012;7:70.

3. Hans CP, Weisenburger DD, Greiner TC, et al. Confirmation of the molecular classification of diffuse large B-cell lymphoma by immunohistochemistry using a tissue microarray. Blood 2004;103:275-82.

4. Murase T, Yamaguchi M, Suzuki R, et al. Intravascular large B-cell lymphoma (IVLBCL): a clinicopathologic study of 96 cases with special reference to the immunophenotypic heterogeneity of CD5. Blood 2007;109:478-85.

5. Matsue K, Abe Y, Narita K, et al. Diagnosis of intravascular large B cell lymphoma: novel insights into clinicopathological features from 42 patients at a single institution over 20 years. Br J Haematol 2019;187:328-36.

6. Zhang Y, Bi L, Qiu Y, et al. Primary pulmonary intravascular large B-cell lymphoma: A report of three cases and literature review. Oncol Lett 2018;15:3610-3.

Cite this article as: Zhu D, Shen Y, Huang J, Zhao L. Intravascular large B-cell lymphoma with diffuse ground glass lesion on chest computed tomography diagnosed using transbronchial lung cryobiopsy: a case report. Transl Cancer Res 2021;10(10):4571-4576. doi: 10.21037/tcr-21-769
7. Souza CA, Quan K, Seely J, et al. Pulmonary intravascular lymphoma. J Thorac Imaging 2009;24:231-3.

8. Ponzoni M, Arrigoni G, Gould VE, et al. Lack of CD 29 (beta1 integrin) and CD 54 (ICAM-1) adhesion molecules in intravascular lymphomatosis. Hum Pathol 2000;31:220-6.

9. Kato M, Ohshima K, Mizuno M, et al. Analysis of CXCL9 and CXCR3 expression in a case of intravascular large B-cell lymphoma. J Am Acad Dermatol 2009;61:888-91.

10. Shiiba M, Izutsu K, Ishihara M. Early detection of intravascular large B-cell lymphoma by (18)FDGPET/CT with diffuse FDG uptake in the lung without respiratory symptoms or chest $\mathrm{CT}$ abnormalities. Asia Ocean J Nucl Med Biol 2014;2:65-8.

11. Tobin-Vealey K, Jain S, Al Diffalha S, et al. Use of Fluorodeoxyglucose-Positron Emission Tomography in the Diagnosis of Intravascular Diffuse Large B-Cell Lymphoma. Fed Pract 2016;33:32S-6S.

12. Colella S, Haentschel M, Shah P, et al. Transbronchial Lung Cryobiopsy in Interstitial Lung Diseases: Best Practice. Respiration 2018;95:383-91.

13. Mehrad M, Colby TV, Rossi G, et al. Transbronchial Cryobiopsy in the Diagnosis of Fibrotic Interstitial Lung Disease. Arch Pathol Lab Med 2020;144:1501-8.

14. Yap E, Low I. Bronchoscopic Transbronchial Cryobiopsy Diagnosis of Recurrent Diffuse Large B-Cell Lymphoma in the Lung: A Promising New Tool? J Bronchology Interv Pulmonol 2017;24:e22-3.

15. Bianchi R, Dubini A, Asioli S, et al. Transbronchial cryobiopsy: an effective tool in the diagnosis of lymphoproliferative disorders of the lung. ERJ Open Res 2020;6:00260-2019. 